\title{
An Insight into the Solar Dynamo Theory
}

\author{
Babu Ram Tiwari* and Mukul Kumar \\ State Key Laboratory of Space Weather, National Space Science Centre \\ Chinese Academy of Sciences, Beijing, China-100190
}

\begin{abstract}
ABSTRA C T
The Sun manifests its magnetic field in form of the solar activities, being observed on the surface of the Sun. The dynamo action is responsible for the evolution of the magnetic field in the Sun. The present article aims to present an overview of the studies have been carried on the theory and modelling of the solar dynamo. The article describes the alpha-omega dynamo model. Generally, the dynamo model involves the cyclic conversion between the poloidal field and the toroidal field. In case of alpha-omega dynamo model, the strong differential rotation generates a toroidal field near the base of the convection zone. On the other hand, the turbulent helicity leads to the generation of the poloidal field near the surface. The turbulent diffusion and the meridional circulation are considered as the two important flux transport agents in this model. The article briefly describes the theory of solar dynamo and mean field dynamo model.
\end{abstract}

Key Words: Solar Dynamo, Mean Field Dynamo Theory, Magnetohydrodynamics, AlphaOmega Dynamo Model.

\section{Introduction}

"Is dynamo action really responsible for the generation of solar magnetic field ?", one of the most coercing questions made by Larmor in 1919 [1], was answered during 1950-1980 [2]. Yes, for a prescribed flow in a body with uniform conductivity, a dynamo action is possible [3]. However, later, the dynamo action was tested in many simple periodic flows [4]. As the next step in dynamo theory, it was found that in a rotating system, the convection-driven turbulence can produce a magnetic field [5],[6]. The next challenge was to identify the motions which are in fact responsible for generating the magnetic field of the Sun and in this direction, the work of Babcock, Leighton and Leighton led to provide a

* Corresponding author email: babu.cas@,outlook.com DOI : https://doi.org/10.21467/ias.3.1.27-36 solar dynamo model [7]-[9]. Stix [10] classified this model in the category of the mean-field models, is considered as a special example of mean-field models.

In the modern era, the objective of the solar dynamo theory is to understand the mechanism for generation of the solar magnetic field by a dynamo. The observations of different type of solar magnetic fields are contributing to enrich our existing knowledge and understanding of solar dynamo. There have been two fundamental aspects of the studies in astrophysics and space physics are (1) the origin of the magnetic fields observed in most observable cosmic objects from planets to galaxies, and (2) their persistence over time [11]. In particular, the explanation of the origin of the magnetic field of the Sun has been at the forefront of the research in magnetohydrodynamics [12]. The magnetic field 
of the Sun is responsible for solar transient activities and tremendous heating of solar corona. All these phenomena have important consequences for the terrestrial environment. They may cause severe magnetic storms leading to problems in the navigation systems of spacecraft orbiting the Earth. They may interrupt the telecommunication system and can affect the power grids. In severe conditions, the human health is also at risk.

This article attempts to describe briefly the general aspects of the basic theory of solar magnetic dynamo, succinctly introducing the theory of mean field dynamo, which has been successful in explaining some of the main features of the solar cycle. Section 2 defines the basic theory of dynamo. Section 3 gives a brief description of mean field dynamo theory along with the alpha-omega dynamo and some examples of the simulated solar cycle using the mean field model. Section 4 contains the concluding remarks.

\section{Basic Theory of Solar Dynamo}

A systematic understanding of the process through which a rotating, convecting, and electrically conducting fluid acts to maintain a magnetic field is known as dynamo theory solar magnetic fields is given by the dynamo theory. It is the process of conversion of kinetic energy into magnetic energy. The currents required for sustainability of the field are being induced by the motion of a fluid [12]. It is accepted that dynamo action gives rise to the magnetic activities, being observed on the Sun. The Magnetohydrodynamics is a combination of Maxwell's equations of electrodynamics, the fluid equations and the Lorentz forces due to electromagnetic fields [13]

Maxwell's equations, the building blocks of electromagnetic theory are considered to be the foundation of dynamo theory. They are as follows-

$$
\begin{aligned}
& \nabla \times \vec{E}=-\frac{\partial \overrightarrow{\mathrm{B}}}{\partial \mathrm{t}} \\
& \nabla \times \vec{B}=\mu_{O} \vec{J}+\mu_{O} \epsilon_{o} \frac{\partial \vec{E}}{\partial t} \\
& \nabla \cdot \vec{B}=0 \\
& \nabla \cdot \vec{E}=\rho / \epsilon_{o}
\end{aligned}
$$

Where $\vec{E}, \vec{B}$, and $\vec{J}$ stands for electric field, magnetic field and current density, respectively. $\mu_{O}$ is the permeability, $\mu_{o}=4 \pi \times 10^{-7}$ in free space, c is the speed of light, $\rho$ is the charge density and $\epsilon_{o}$ is the permitivity of free space. In free space $\epsilon_{o}=\left(\mu_{o} c^{2}\right)^{-1}$.

Equation (2.1) states that a time-varying magnetic field produces an electric field. In an electrically conducting body, this electric field drives a current, which is the basis of dynamo action. Equation (2.3) states that no magnetic monopole exists in nature. However, equation (2.4) states that there exist electric monopoles from which electric field originate. These are electrons and protons. Maxwell's equations are relativistically invariant, but in MHD framework fluid velocity is considered to be very small as compared to the speed of light. This allows us to discard the term $\mu_{\mathrm{o}} \epsilon_{\mathrm{o}} \frac{\partial \overrightarrow{\mathrm{E}}}{\partial \mathrm{t}}$ and equation (2.2) becomes:

$\nabla \times \overrightarrow{\mathrm{B}}=\mu_{\mathrm{O}} \overrightarrow{\mathrm{J}}$

Equation (2.5) is known as the Ampere's law or pre-Maxwell equation.

In a moving frame, $\vec{E}$ must be replaced by $\overrightarrow{\mathrm{E}}+$ $\overrightarrow{\mathrm{u}} \times \overrightarrow{\mathrm{B}}$ while $\overrightarrow{\mathrm{J}}$ stays the same. Hence, in MHD Ohm's law:

$$
\overrightarrow{\mathrm{J}}=\sigma(\overrightarrow{\mathrm{E}}+\overrightarrow{\mathrm{u}} \times \overrightarrow{\mathrm{B}})
$$

The magnetic diffusivity is defined as $\eta=\frac{1}{\mu_{\mathrm{o}} \sigma}$, (with dimensions metre $^{2} /$ second). Hence, the poor conductors have large magnetic diffusivity $\eta$ with the perfectly conducting limit as $\eta \rightarrow 0$. Substituting equation (2.6) in equation (2.5), and taking curl on both sides, we get:

$$
\nabla \times \nabla \times \vec{B}=\mu_{o} \sigma(\nabla \times \vec{E}+\nabla \times \vec{u} \times \vec{B})
$$

Using vector identity,

$$
\begin{aligned}
& \nabla \times \nabla \times \overrightarrow{\mathrm{B}}=\nabla(\nabla \cdot \overrightarrow{\mathrm{B}})-(\nabla \cdot \nabla) \overrightarrow{\mathrm{B}} \\
& \text { i.e. } \nabla(\nabla \cdot \vec{B})-\nabla^{2} \vec{B}=\mu_{o} \sigma\left(-\frac{\partial \vec{B}}{\partial t}+\nabla \times \vec{u} \times \vec{B}\right)
\end{aligned}
$$$$
\text { Since } \nabla \cdot \vec{B}=0
$$$$
\left.\frac{\partial \overrightarrow{\mathrm{B}}}{\partial \mathrm{t}}=\nabla \times \overrightarrow{(\mathrm{u}} \times \overrightarrow{\mathrm{B}}\right)+\eta \nabla^{2} \overrightarrow{\mathrm{B}}
$$

Equation (2.7), the induction equation is a key equation in MHD, since it contains the Faraday's law of electromagnetic induction. If $\mathrm{U}, \mathrm{B}$ and $\mathrm{L}$ stand for the typical values of velocity, magnetic field and length scale respectively then the two terms on the RHS of equation (2.7) are of the 
Tiwari et al., Int. Ann. Sci.; Vol. 3 Issue 1, pp: 27-36, 2017

order $\mathrm{UB} / \mathrm{L}$ and, $\eta \mathrm{B} / \mathrm{L}^{2}$. The ratio of these two terms gives a dimensionless number, which is called the magnetic Reynolds number, given by

$\mathrm{R}_{\mathrm{m}}=\frac{\mathrm{UB} / \mathrm{L}}{\eta \mathrm{B} / \mathrm{L}^{2}}=\frac{\mathrm{UL}}{\eta}$

The value of $R_{m}$ is found to be higher $\left(R_{m}>>1\right)$ for astrophysical systems and lower $\left(R_{m} \ll 1\right)$ for the laboratory. Hence, the magnetic fields show different behaviour in laboratory plasmas and astrophysical plasmas. For example, it is not possible to develop a laboratory analogue of the Sun's self-sustaining magnetic field. A dynamo mechanism has been demonstrated in the laboratory by [14]. In an astrophysical system, if $R_{m} \gg 1$, the diffusion term in equation (2.7) can be considered as negligible compared to its preceding term. This is the frozen flux limit, so called because the flux through any closed loop that is the surface integral B over the loop, remains fixed as the loop moves around with the fluid velocity. That means the magnetic field is frozen in the plasma and moves with it. The fluxfreezing theorem was coined by Hannes Alfven in 1942 and so it is often referred as Alfven theorem of flux-freezing [15]. For $R_{m} \ll 1$, the induction term can be neglected from equation (2.7) and only diffusion term will survive. If there is no fluid motion to maintain the dynamo, the field diffuses away. More precisely, if there is no field at infinity it diffuses away to zero, but if a conductor is immersed in a uniform field, the field inside the conductor eventually becomes uniform [16].

The estimated numerical values for relevant nondimensional parameters at the convection zone as well as at the photosphere has been given by [17]. These values are displayed in Table 2.1.

There are two important restrictions on the types of solutions that can sustain the self-exciting mechanism. The first of these is known as the Cowling theorem (1933) [18] revealing that a magnetic field with rotational symmetry cannot be maintained by the dynamo action [18], [19]. Accordingly, the solar magnetic field is nonaxisymmetric. In 1961 Babcock presented a model of solar cycle where it was shown from a typical dipole magnetic field (or poloidal) is generated one component in the azimuthal direction (or toroidal) product of the differential rotation. This phenomenon is called $\Omega$ - the effect is due to the magnetic diffusivity in the convective region is very small, "frozen" [20] state of magnetic field lines.

Table 2.1: Dimensionless parameters characterizing convection and dynamo action [17]

\begin{tabular}{|l|l|l|l|}
\hline \multicolumn{4}{|l|}{ Base of convection zone Photosphere } \\
\hline $\begin{array}{l}\text { Reynolds } \\
\text { number }\end{array}$ & $\mathrm{R}_{\mathrm{e}}=\mathrm{UL} / \nu$ & $10^{13}$ & $10^{12}$ \\
\hline $\begin{array}{l}\text { Magnetic } \\
\text { Reynolds } \\
\text { number }\end{array}$ & $\mathrm{R}_{\mathrm{m}}=\mathrm{UL} / \eta$ & $10^{10}$ & $10^{6}$ \\
\hline Prandtl number & $\mathrm{P}_{\mathrm{r}}=v / k$ & $10^{-7}$ & $10^{-7}$ \\
\hline $\begin{array}{l}\text { Magnetic } \\
\text { Prandtl number }\end{array}$ & $\mathrm{P}_{\mathrm{m}}=v / \eta$ & $10^{-3}$ & $10^{-6}$ \\
\hline Rossby number & $\mathrm{R}_{\mathrm{o}}=\mathrm{U} / 2 \Omega \mathrm{L}$ & $0.1-1$ & $10^{-3}-0.4$ \\
\hline $\begin{array}{l}\text { Coriolis number } \\
\mathrm{C}_{\mathrm{o}}=2 \Omega \mathrm{L} / \mathrm{U}\end{array}$ & 15 & $2 \times 10^{-3}$ \\
\hline Mach number & $\mathrm{M}_{\mathrm{a}}=\mathrm{U} / \mathrm{C}_{\mathrm{s}}$ & $10^{-4}$ & 1 \\
\hline Plasma $\beta$ & $\beta=2 \mu_{\mathrm{o}} / \mathrm{B}^{2}$ & $10^{5} \ldots 10^{7}$ & 1 \\
\hline
\end{tabular}

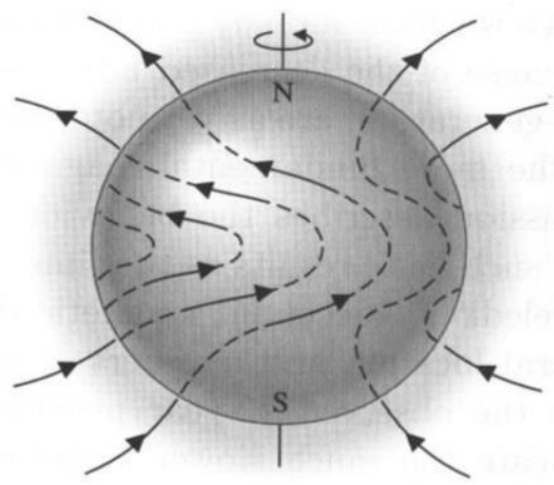

(a)

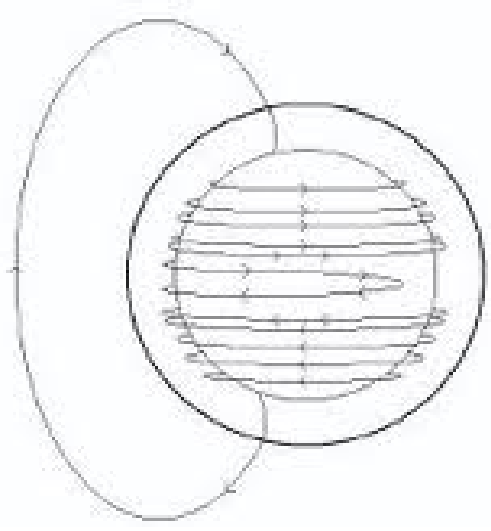

(b)

Figure 2.1: The lines of a dipolar magnetic field originally coiling in azimuthal due to differential rotation (a) at the beginning of the process (b) after several rotations, one can observe the generation of a strong toroidal field [7] [23]

Figure 2.1 demonstrates the differential rotation of the Sun. The other restriction, according to 
Elsasser, the differential rotation alone is not capable of driving a dynamo in a sphere unless the magnetic diffusivity is constant on spherical surfaces [21], [22]. Because of these restrictions, a complex three-dimensional structure of the generated magnetic field and non-axisymmetric structure of driving velocity field are required for a working dynamo [23]. As the rotation of the Sun is differential, the angular velocity at the equator is found to be $20 \%$ faster as compared to that as of the poles. The differential rotation and convective flows are the two vital components of the self-exciting solar dynamo action [23]. The generation and amplification of the magnetic field from a meridional field with radial and latitudinal components is caused by the shearing effect of latitudinal and radial gradients of the angular velocity of rotation which arises because of flux freezing.

The Parker loop (shown in Figure 2.2) is considered as one of the most suitable convection-based models for the above process. This explains how the magnetic field lines get twisted by the action of the Coriolis force on the flow and then how the convective upflows and downflows generate the meridional flux loops through these twisted field lines. This gives the opposite senses of twisting by the Coriolis force in two different hemispheres. For the pressure stratification of the convective zone, upflows expand and downflows contract [24]. A simple explanation for the reversals of the large-scale meridional field of the Sun is given by the Parker loop.

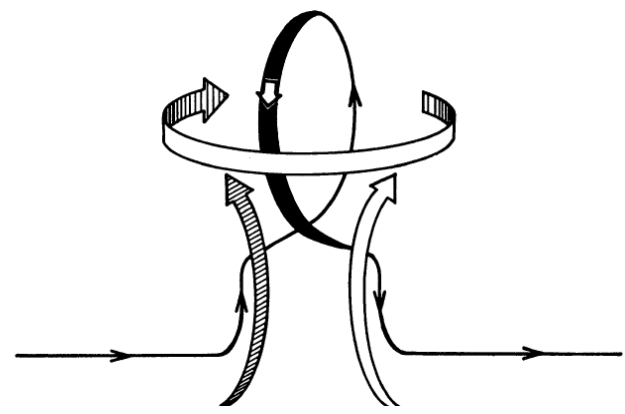

Figure 2.2: Sketch of the Parker loop: It shows that because of the action of the Coriolis force in a rotating system an expanding convective upflow gets into the vortical motion. It further shows that a magnetic field line which is frozen into the plasma get twisted into a loop with magnetic field components perpendicular to the plane of projection [24].

\section{Mean Field Dynamo}

The mean field dynamo theory presents the theoretical explanations of the large-scale magnetic activity in cosmic bodies [28]-[31]. The interaction with the large-scale as well as smallscale components of the magnetic fields is necessary to determine the large-scale solar magnetic activities. Therefore, mean field dynamo theory gives the interaction between the mean and fluctuating parts of the magnetic fields. The mean field dynamo theory has its several application, e.g. the use of the $\alpha$-effect term in the induction euation of solar and stellar models. In the theory of 'Mean-Field Electrodynamics' (MFE), the mathematical treatement of the 'Parker mechanism' has been given by Steenbeck et al. [6]. It explains that how an avarage magnetic field evolute in a turbulent flow of an electrically conducting fluid. The mean-field theory is based on the idea of spliting the magnetic field and the flow into mean and fluctuating parts, so

$\overrightarrow{\mathrm{U}}(\overrightarrow{\mathrm{x}}, \mathrm{t})=\overrightarrow{\mathrm{U}}_{\mathrm{o}}(\overrightarrow{\mathrm{x}}, \mathrm{t})+\overrightarrow{\mathrm{u}}(\overrightarrow{\mathrm{x}}, \mathrm{t}), \overrightarrow{\mathrm{U}}_{\mathrm{o}}=\langle\overrightarrow{\mathrm{U}}\rangle$

Where the total velocity field $\vec{U}(\vec{x}, t)$ as a known function of position and time while $\overrightarrow{\mathrm{u}}(\overrightarrow{\mathrm{x}}, \mathrm{t})$ is the random or fluctuating part.

$\vec{B}(\vec{x}, t)=\vec{B}_{o}(\vec{x}, t)+\vec{b}(\vec{x}, t), \vec{B}_{o}=\langle\vec{B}\rangle$

The mean part considered the large-scale average field and velocity while the fluctuating part is considered as a small scale turbulent contribution. We need an averaging procedure is such a way that the fluctuating quantities on their own average to zero, but products of fluctuating quantities can have a non-zero average. So,

$\langle\overrightarrow{\mathrm{u}}\rangle=\langle\overrightarrow{\mathrm{b}}\rangle=0$

The averaging process must be defined keeping in mind that it must obey the averaging rules. The sum of the average of a number of terms must be the average of their sum. Their averaging should be commutative with their differentiation with respect to space as well as with time. However, averaging an averaged quantity leaves it unchanged namely,

$\langle\lambda \mathrm{P}+\mu \mathrm{Q}\rangle=\lambda\langle\mathrm{P}\rangle+\mu\langle\mathrm{Q}\rangle$

Where $\mathrm{P}$ and $\mathrm{Q}$ are scalar functions of position and time respectively. However, $\lambda$ and $\mu$ in the equation are the arbitrary numbers.

$\langle\cdot\rangle$ Commutes with $\partial_{\mathrm{t}}, \nabla$ and $\int \mathrm{dt}$; 
Tiwari et al., Int. Ann. Sci.; Vol. 3 Issue 1, pp: 27-36, 2017

$\langle\mathrm{PQ}\rangle=\langle\mathrm{P}\rangle\langle\mathrm{Q}\rangle$,

The above property holds true for the statistically independent fluctuating elements in P and Q and the average must be linear.

$\langle P\langle Q\rangle\rangle=\langle P\rangle\langle Q\rangle$,

That is (3.5) implies (3.6) but not vice-versa. Relations (3.4), (3.5), (3.6), plus the trivial relation $\langle\mu\rangle=\mu$, constitute the Reynolds relations [25], [26].

Substituting the equations (3.1) and (3.2) into the induction equation (2.7), we get;

$$
\begin{gathered}
\partial_{\mathrm{t}}\left(\overrightarrow{\mathrm{B}}_{\mathrm{o}}+\overrightarrow{\mathrm{b}}\right)=\nabla \times\left[\left(\overrightarrow{\mathrm{U}}_{\mathrm{o}}+\overrightarrow{\mathrm{u}}\right) \times\left(\overrightarrow{\mathrm{B}}_{\mathrm{o}}+\overrightarrow{\mathrm{b}}\right)\right] \\
+\eta \nabla^{2}\left(\overrightarrow{\mathrm{B}}_{\mathrm{o}}+\overrightarrow{\mathrm{b}}\right) \\
=\nabla \times \overrightarrow{\mathrm{U}}_{\mathrm{o}} \times\left(\overrightarrow{\mathrm{B}}_{\mathrm{o}}+\overrightarrow{\mathrm{b}}\right)+\nabla \times \overrightarrow{\mathrm{u}} \times\left(\overrightarrow{\mathrm{B}}_{\mathrm{o}}+\overrightarrow{\mathrm{b}}\right)+ \\
\eta \nabla^{2} \overrightarrow{\mathrm{B}}_{\mathrm{o}}+\eta \nabla^{2} \overrightarrow{\mathrm{b}}
\end{gathered}
$$

Taking average of the resulting equation yields evolution equation for the mean field

$$
\left.\begin{array}{l}
\partial_{\mathrm{t}} \overrightarrow{\mathrm{B}}_{\mathrm{o}} \\
\eta \nabla^{2} \overrightarrow{\mathrm{B}}_{\mathrm{o}}
\end{array}=\nabla \times \overrightarrow{\mathrm{U}}_{\mathrm{o}} \times \overrightarrow{\mathrm{B}}_{\mathrm{o}}\right)+\langle\nabla \times(\overrightarrow{\mathrm{u}} \times \overrightarrow{\mathrm{b}})\rangle+
$$

Here, the term which contains the products of a mean and a fluctuating quantity average to zero. However, the term which involves the products of fluctuating quantities can have a non-zero mean part. A non-zero mean e.m.f can be obtained by systematically aligning the fluctuating field $\overrightarrow{\mathrm{b}}$ and the fluctuating turbulent part of the velocity field $\overrightarrow{\mathrm{u}}$.

$\vec{\varepsilon}=\langle\overrightarrow{\mathrm{u}} \times \overrightarrow{\mathrm{b}}\rangle$

The new mean e.m.f. term in the mean induction equation (3.8) displays the no more applicability of the Cowling's theorem. So, the simple axisymmetric dynamos as solutions can be found [27]. It is visible that most of the work carried out on dynamo theory has utilised the mean field equations with a non-zero mean e.m.f. The mean e.m.f. term developed the poloidal field from toroidal field, and then closes the loop. The Figure 3.1, describes the mechanism of the generation of toroidal field fro poloidal field and vice-versa; However, the Figure 3.2 explains that how a toroidal field is being generated from the poloidal field via the turbulent alpha-effect mechanism.

Now, subtracting the mean-field equation (3.8) from the full induction equation (3.7), we obtain the equation for the fluctuating field $\vec{b}$, $\partial_{\mathrm{t}} \overrightarrow{\mathrm{b}}=\nabla \times\left(\overrightarrow{\mathrm{U}}_{\mathrm{o}} \times \overrightarrow{\mathrm{b}}\right)+\nabla \times\left(\overrightarrow{\mathrm{u}} \times \overrightarrow{\mathrm{B}}_{\mathrm{o}}\right)+\nabla \times$

$[(\overrightarrow{\mathrm{u}} \times \overrightarrow{\mathrm{b}})-\langle\overrightarrow{\mathrm{u}} \times \overrightarrow{\mathrm{b}}\rangle]+\eta \nabla^{2} \overrightarrow{\mathrm{b}}$

By considering $\overrightarrow{\mathrm{u}}$ to be isotropic, $\overrightarrow{\mathrm{U}}_{\mathrm{o}}$ to be constant, the term $\nabla \times\left(\vec{U}_{0} \times \vec{b}\right)$ can be omitted from the equation (3.10). For $\vec{b} \ll \vec{B}_{0}$, the terms $(\overrightarrow{\mathrm{u}} \times \overrightarrow{\mathrm{b}})-\langle\overrightarrow{\mathrm{u}} \times \overrightarrow{\mathrm{b}}\rangle$ are negligible as compared to $\overrightarrow{\mathrm{u}} \times \overrightarrow{\mathrm{B}}_{\mathrm{o}}$. This hypothesis is called the First Order Smoothing Approximation (FOSA) or SecondOrder Correlation Approximation (SOCA), where all the correlations higher than the second order in fluctuations are neglected.

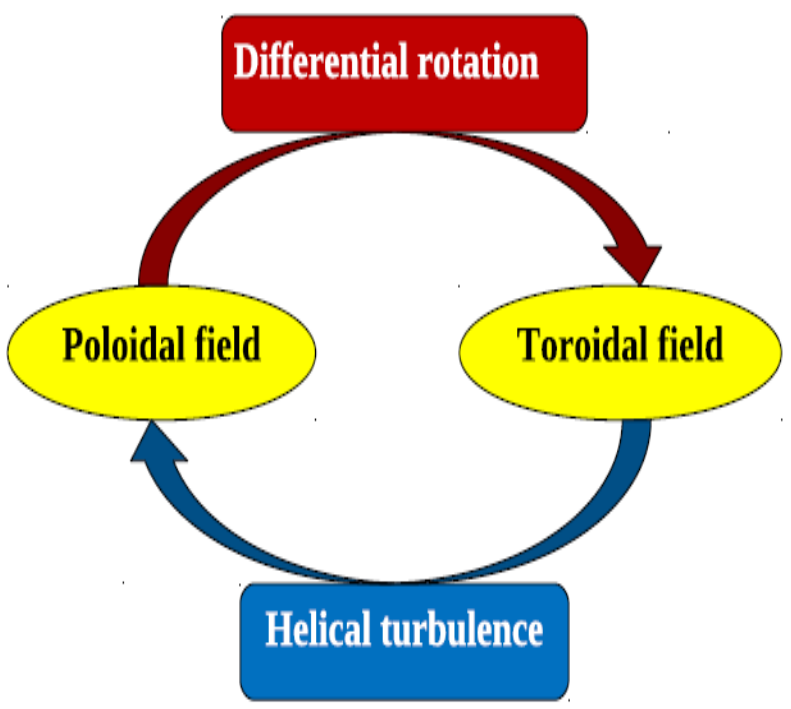

Figure 3.1: The Cartoon showing the dynamo model in a nutshell

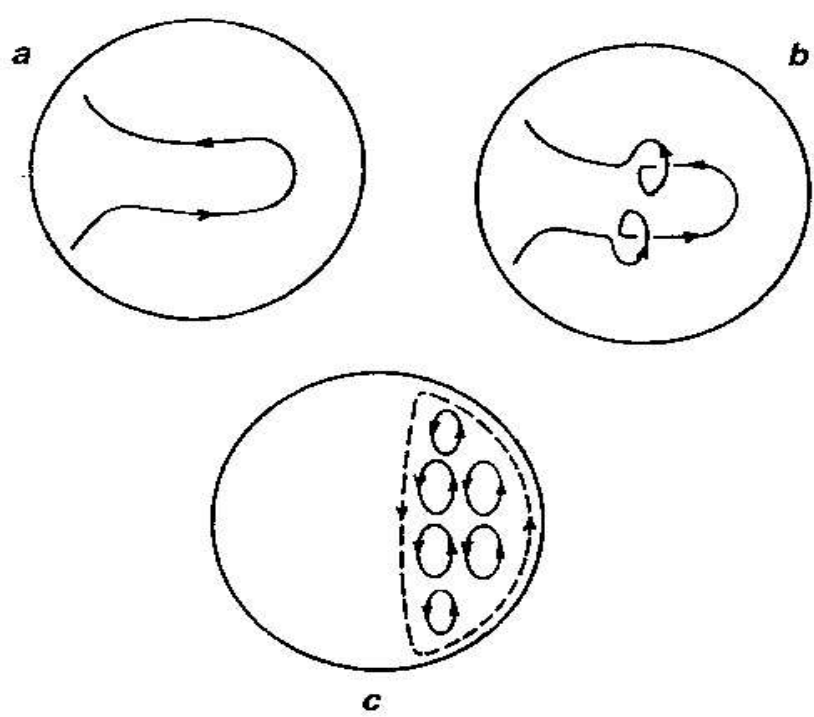

Figure 3.2: The turbulent $\alpha$-effect mechanism of producing the poloidal field from toroidal field [16] 
We assume that $R_{m} \gg 1$ in the astrophysical system so that we may also neglect the last term. Then, equation (3.10) is of the form:

$\partial_{\mathrm{t}} \overrightarrow{\mathrm{b}}=\nabla \times\left(\overrightarrow{\mathrm{u}} \times \overrightarrow{\mathrm{B}}_{\mathrm{o}}\right)$

$\frac{\partial \vec{b}}{\partial t}=\left(\overrightarrow{\mathrm{B}}_{\mathrm{o}} \cdot \nabla\right) \overrightarrow{\mathrm{u}}-(\overrightarrow{\mathrm{u}} \cdot \nabla) \overrightarrow{\mathrm{B}}_{\mathrm{o}}$

For simplicity, if the fluid is incompressible, then $\nabla \cdot \vec{u}=0$ and $\nabla \cdot \vec{B}_{o}=0$. So, integrating Lagrangian long time, $\vec{x}^{\prime}=\vec{x}\left(t^{\prime}\right)$ for the spatiotemporal mean field:

$\overrightarrow{\mathrm{b}}(\overrightarrow{\mathrm{x}}, \mathrm{t})=\overrightarrow{\mathrm{b}}(\overrightarrow{\mathrm{x}},-\infty)+\int_{-\infty}^{\mathrm{t}} \frac{\partial \overrightarrow{\mathrm{b}}}{\partial \mathrm{t},} \mathrm{dt}^{\prime}$

Here, the lower limit equal to $-\infty$ because of the short correlation time that makes the values of $\mathrm{t}^{\prime}$ significantly smaller than $t$ irrelevant.

$\vec{b}(\vec{x}, t)=\vec{b}(\vec{x},-\infty)+\int_{-\infty}^{t} d t^{\prime}\left[\left(\vec{B}_{o}\right.\right.$.

$\left.\nabla) \overrightarrow{\mathrm{u}}\left(\overrightarrow{\mathrm{x}}^{\prime}, \mathrm{t}^{\prime}\right)-\overrightarrow{\mathrm{u}}\left(\overrightarrow{\mathrm{x}}^{\prime}, \mathrm{t}^{\prime}\right) \cdot \nabla \overrightarrow{\mathrm{B}}_{\mathrm{o}}\right]$

So, the turbulent e.m.f. is

$$
\begin{aligned}
\vec{\varepsilon}=\langle\overrightarrow{\mathrm{u}} \times \overrightarrow{\mathrm{b}}\rangle= & \langle\overrightarrow{\mathrm{u}}(\overrightarrow{\mathrm{x}}, \mathrm{t}) \times \overrightarrow{\mathrm{b}}(\overrightarrow{\mathrm{x}},-\infty)\rangle \\
& +\int_{-\infty}^{\mathrm{t}} \mathrm{dt}^{\prime}\langle\overrightarrow{\mathrm{u}}(\overrightarrow{\mathrm{x}}, \mathrm{t}) \\
& \times\left[\left(\overrightarrow{\mathrm{B}}_{\mathrm{o}} \cdot \nabla\right) \overrightarrow{\mathrm{u}}\left(\overrightarrow{\mathrm{x}}^{\prime}, \mathrm{t}^{\prime}\right)-\overrightarrow{\mathrm{u}}\left(\overrightarrow{\mathrm{x}}^{\prime}, \mathrm{t}^{\prime}\right)\right. \\
& \left.\left.\cdot \nabla \overrightarrow{\mathrm{B}}_{\mathrm{o}}\right]\right\rangle
\end{aligned}
$$

Here $\overrightarrow{\mathrm{u}}(\overrightarrow{\mathrm{x}}, \mathrm{t})$ may not be correlated with $\overrightarrow{\mathrm{b}}(-\infty)$, the first term vanishes. So,

$\vec{\varepsilon}=\int_{-\infty}^{\mathrm{t}} \mathrm{dt}^{\prime}\left\langle\overrightarrow{\mathrm{u}}(\overrightarrow{\mathrm{x}}, \mathrm{t}) \times\left[\left(\overrightarrow{\mathrm{B}}_{\mathrm{o}} \cdot \nabla\right) \overrightarrow{\mathrm{u}}\left(\overrightarrow{\mathrm{x}}^{\prime}, \mathrm{t}^{\prime}\right)-\right.\right.$

$\left.\left.\overrightarrow{\mathrm{u}}\left(\overrightarrow{\mathrm{x}}^{\prime}, \mathrm{t}^{\prime}\right) \cdot \nabla \overrightarrow{\mathrm{B}}_{\mathrm{o}}\right]\right\rangle$

Acting as a source term, $\nabla \times\left(\overrightarrow{\mathrm{u}} \times \overrightarrow{\mathrm{B}}_{\mathrm{o}}\right)$ in (3.10), give rise to the generation of fluctuating field $\vec{b}$. If we take $\vec{b}=0$ at $\mathrm{t}=0, \overrightarrow{\mathrm{B}}_{\mathrm{o}}$ and $\overrightarrow{\mathrm{b}}$ as well as $\varepsilon$ and $\vec{B}_{o}$ are found to be linearly correlated. This linear relationship may be represented as:

$\vec{\varepsilon}_{\mathrm{i}}=\alpha_{\mathrm{ij}} \overrightarrow{\mathrm{B}}_{\mathrm{oj}}+\beta_{\mathrm{ijk}} \frac{\partial \overrightarrow{\mathrm{B}}_{\mathrm{oj}}}{\partial \mathrm{x}_{\mathrm{k}}}+\gamma_{\mathrm{ijkl}} \frac{\partial^{2} \overrightarrow{\mathrm{B}}_{\mathrm{oj}}}{\partial \mathrm{x}_{\mathrm{k}} \partial \mathrm{x}_{\mathrm{l}}}+$, (3.16)

Terms containing time derivatives $\frac{\partial \overrightarrow{\mathrm{B}}_{\mathrm{oj}}}{\partial \mathrm{t}}, \frac{\partial^{2} \overrightarrow{\mathrm{B}}_{\mathrm{oj}}}{\partial \mathrm{t}^{2}}, \ldots$ may also appear in the expression for $\overrightarrow{\boldsymbol{\varepsilon}}$. Using the equation (3.8), such terms can be replaced with the terms containg only space derivatives. Higher order terms have been neglected and this series will rapidly be convergent [28]. In equation (3.16) $\alpha$ and $\beta$ are 2nd and 3rd rank tensors respectively and that depend on $\vec{u}$ and $\vec{U}_{\mathbf{o}}$. The pseudo-tensor coefficients have the following notation (summation convention)

$\alpha_{i j} \vec{B}_{o j}=\sum_{j=1}^{3} \alpha_{i j} \vec{B}_{o j}$

$\beta_{\mathrm{ijk}} \frac{\partial \overrightarrow{\mathrm{B}}_{\mathrm{oj}}}{\partial \mathrm{x}_{\mathrm{k}}}=\sum_{\mathrm{j}=1}^{3} \sum_{\mathrm{k}=1}^{3} \beta_{\mathrm{ijk}} \frac{\partial \overrightarrow{\mathrm{B}}_{\mathrm{oj}}}{\partial \mathrm{x}_{\mathrm{k}}}$

We can attain the large-scale dynamo mechanism if we consider an isotropic velocity field $\vec{u}$. In this case $\alpha$ and $\beta$ must be isotropic and invariant under rotation, so that $\alpha_{\mathrm{ij}}=\alpha \delta_{\mathrm{ij}}$ and $\beta_{\mathrm{ijk}}=$ $-\beta \epsilon_{\mathrm{ijk}}$, and where $\delta_{\mathrm{ij}}$ is the Kronecker tensor and $\epsilon_{\mathrm{ijk}}$ the Levi-Civita tensor which has a permutation relation

$$
\begin{aligned}
& \epsilon_{\mathrm{ijk}} \\
& =\left\{\begin{array}{c}
0 \text { if } \mathrm{i}=\mathrm{j}, \mathrm{j}=\mathrm{k}, \text { or } \mathrm{k}=\mathrm{i} \\
+1 \text { if }(\mathrm{i}, \mathrm{j}, \mathrm{k}) \in\{(1,2,3),(2,3,1),(3,1,2)\} \\
-1 \text { if }(\mathrm{i}, \mathrm{j}, \mathrm{k}) \in\{(1,3,2),(3,2,1),(2,1,3)\}
\end{array}\right\}
\end{aligned}
$$

Hence the equation (3.16) becomes

$$
\vec{\varepsilon}=\alpha \overrightarrow{\mathrm{B}}_{\mathrm{o}}-\beta \nabla \times \overrightarrow{\mathrm{B}}_{\mathrm{o}}
$$

Where $\alpha$ is a pseudo-scalar, dependent on the set of the right-handed set or left-handed coordinate system (i. e. $\alpha \rightarrow-\alpha$ e.g $x \rightarrow-x$ ) and $\beta$ is a true scalar.

Substituting equation (3.17) for the mean electromotive force with the mean field equation (3.8) gives

$$
\partial_{\mathrm{t}} \overrightarrow{\mathrm{B}}_{\mathrm{o}}=\nabla \times\left(\overrightarrow{\mathrm{U}}_{\mathrm{o}} \times \overrightarrow{\mathrm{B}}_{\mathrm{o}}\right)+\nabla \times\left[\alpha \overrightarrow{\mathrm{B}}_{\mathrm{o}}-\beta(\nabla \times\right.
$$$$
\left.\left.\overrightarrow{\mathrm{B}}_{\mathrm{o}}\right)\right]+\eta \nabla^{2} \overrightarrow{\mathrm{B}}_{\mathrm{o}}
$$

Equation (3.18) is known as the mean-field dynamo equation and, provided $\overrightarrow{\mathrm{U}}_{\mathrm{o}}, \alpha, \beta$ and $\eta$ are known, it can be solved for the mean field.

$\partial_{\mathrm{t}} \overrightarrow{\mathrm{B}}_{\mathrm{o}}=\nabla \times\left(\overrightarrow{\mathrm{U}}_{\mathrm{o}} \times \overrightarrow{\mathrm{B}}_{\mathrm{o}}\right)+\nabla \times \alpha \overrightarrow{\mathrm{B}}_{\mathrm{o}}+(\eta+$

B) $\nabla^{2} \overrightarrow{\mathrm{B}}_{\mathrm{o}}$

This Equation is known as dynamo equation and has to be solved to understand the generation of magnetic field. The physical meaning of the term " $\alpha$-term" that it drives a mean current that is parallel or anti-parallel to the mean magnetic field. This idea was proposed by Parker in 1955 [5].

The physical significance of the " $\beta$-term" seems more clear when $\beta$ is taken to be constant. Since the magnetic field is solenoidal $\left(\nabla \cdot \vec{B}_{o}=0\right), \beta$-term can be written as $\beta \nabla^{2} \vec{B}_{o}$. It can be interpreted as 
Tiwari et al., Int. Ann. Sci.; Vol. 3 Issue 1, pp: 27-36, 2017

eddy diffusivity. In practice, $\eta$ and $\beta$ are combined into a single effective magnetic diffusivity [29].

$\eta_{T}=\eta+\beta$,

Where $\eta_{T}$ is called turbulent diffusivity which in general is much greater than $\eta$. Higher the value of turbulent diffusivity means that the turbulence mixes the magnetic field up to reduce its effective characteristic length-scale down to diffusive scales [30]. We have the terms $\alpha$ and $\beta$ appearing in Equation (3.17) are isotropic and homogeneous. The $\vec{B}_{o}$ trivial integral because not vary with the time of turbulent. Under the first order smoothing approximation (FOSA), the resolution of the Equation (3.15) and comparing with Equation (3.17), we have the relation:

$\alpha=\frac{-1}{3} \int_{-\infty}^{t} d t^{\prime}\left\langle\vec{u} \cdot \nabla \times \vec{u}^{\prime}\right\rangle=\frac{-1}{3} \tau_{\alpha}\langle\vec{u} \cdot \nabla \times$

$\left.\vec{u}^{\prime}\right\rangle=\frac{-1}{3} \tau_{\alpha} H^{k}$

$\beta=\frac{1}{3} \int_{-\infty}^{t} d t^{\prime}\left\langle\vec{u} \cdot \vec{u}^{\prime}\right\rangle=\frac{1}{3} \tau_{\beta}\left\langle u^{2}\right\rangle=\frac{1}{3} \tau_{\beta} E^{k}$

Where $H^{k}$ is known as the kinetic helicity, which results from a net correlation between velocity and vorticity and $\tau_{\alpha} \simeq \tau_{\beta}$ is the correlation time of the turbulent motion. From (3.21), it follows that $\alpha$ is a measure of average helical motion in the fluid. If an element of fluid rises and rotates about the vertical, the flow is called helicity. It describes the production of the poloidal component from the toroidal component by helical turbulent. In 1955 Parker gave a simplified physical explanation of the $\alpha$-effect [5]. The term $E^{k}$ represents the turbulent kinetic energy.

The positive quantity $\beta$ in Equation (3.22) called the turbulent diffusivity. It signifies the enhanced effective diffusion of the mean magnetic field due to the transport of magnetic field lines by the perturbing velocity field and the creation of a small-scale structure and dissipation through the development of a turbulent cascade [23].

\subsection{The $\alpha \Omega$ Dynamo Model}

The solar and stellar dynamos modelling has been based on mean field theory [31], [12]. The application of mean field dynamo equation cannot be justified because there is no separation in length-scales between the mean and fluctuating parts of the magnetic field in the Sun [32]. The mean field dynamo models describe the evolution of the large-scale field by averaging over the turbulent motion. It holds the essential physics of the process, and mean field $(\alpha \Omega)$ dynamo models allow us to explore generic properties of magnetic fields in the stars like the Sun [33], [34].

The physical processes contained within the mean field dynamo equation is described by a simple axisymmetric model in spherical geometry. We can make the decomposition of the mean magnetic fields and velocity fields into poloidal and toroidal parts,

$\vec{B}=\vec{B}_{P}+e_{\varnothing} B_{\emptyset}$,

$\vec{B}_{P}=\nabla \times A e_{\emptyset}$,

$\vec{U}=\vec{U}_{P}+r \Omega e_{\emptyset}$

Where $e_{\varnothing}$ is the unit vector in the $\emptyset$ direction and $\Omega(r, \varnothing)$, the local angular velocity in cylindrical polar coordinates $(r, \varnothing, z)$. Where $A$ is the vector potential of $\vec{B}_{P}$, the mean poloidal magnetic field [26].

Substituting Equation (3.1.1) in mean field induction Equation (3.20) and separating poloidal and toroidal parts, we get:

$\partial_{t} A+\frac{1}{r} \vec{U}_{P} \cdot \nabla r A=\alpha B_{\varnothing}+\eta_{T}\left(\nabla^{2}-\frac{1}{r^{2}}\right) A$

$\partial_{t} B_{\emptyset}+r \vec{U}_{P} \cdot \nabla \frac{1}{r} B_{\emptyset}=r \vec{B}_{P} \cdot \nabla \vec{\Omega}+e_{\emptyset} \cdot(\nabla \times$

$\left.\alpha \vec{B}_{P}\right)+\eta_{T}\left(\nabla^{2}-\frac{1}{r^{2}}\right) B_{\emptyset}$

These linear mean field (or $\alpha \Omega$ ) dynamo Equations have been solved in Cartesian and spherical geometries and for many assumed spatial distributions of $\alpha, \Omega$ and $\eta_{T}$. Equation (3.1.2) if $\alpha=0$, it can be seen that the Equation for $\mathrm{A}$ is of advection-diffusion type. Also, $\mathrm{A} \rightarrow 0$ $\Rightarrow \vec{B}_{P} \rightarrow 0 \Rightarrow$ Eq. (3.1.3) term advectiondiffusion and $B_{\emptyset} \rightarrow 0$.Therefore, $\alpha \neq 0$ is the necessary condition for dynamo. In equation (3.1.3), $r \vec{B}_{P} \cdot \nabla \vec{\Omega}$ i.e. $\Omega$-effect, the toroidal field can be generated from poloidal field. Where, $\nabla \times \alpha \vec{B}_{P} \simeq \alpha \vec{\jmath}$ is the $\alpha$-effect, corresponds to the torsional motion.

Differential rotation converts $\vec{B}_{P}$ into $B_{\varnothing}$, toroidal, and the $\alpha$ effect generates $\vec{B}_{P}$ from $B_{\emptyset}$, 
and vice-versa. Here, the turbulent diffusion time scale, $\tau_{d}=\frac{R^{2}}{\eta_{T}}$.

The linear growth rate of the mean magnetic field depends on a dimensionless combination of the parameters occurring in Equation (3.1.3), the dynamo number, $\mathrm{D}$ is defined as:

$\mathrm{D}=C_{\alpha} C_{\Omega} \equiv \frac{\alpha_{0} R}{\eta_{T}} \frac{\Delta \Omega R^{2}}{\eta_{T}}=\frac{\alpha_{o} \nabla \Omega R^{3}}{\eta_{T}{ }^{2}}$

Where, $C_{\alpha}=\frac{\alpha_{0} R}{\eta_{T}}$ and $C_{\Omega}=\frac{\Delta \Omega R^{2}}{\eta_{T}}$ represent turbulent magnetic Reynolds numbers for the $\alpha$ effect and differential rotation respectively, $\mathrm{R}$ is a typical length scale of dynamo (radius of sphere or disc) and $\Delta \Omega$ are the typical difference in rotation rate within the dynamo region (radial variation of $\Omega$ ), $\alpha_{0}$ is the maximum of $\alpha$. Also, the term, $\eta_{T}=\eta+\beta$ is a turbulent resistivity plus microscopic resistivity.

If $C_{\Omega} \gg C_{\alpha}, \alpha$-effect is ignored in the equation (3.1.3)) but not in (3.1.2), this results the class of the dynamo model called $\alpha \Omega$ dynamo. The rotational shear is the main source of the toroidal component and $\alpha$-effect is the source of only the poloidal component. Therefore, the solar dynamo is believed to be of $\alpha \Omega$ dynamo.

If $C_{\alpha} \gg C_{\Omega}$, the dynamo is of the $\alpha^{2}$ type. The $\alpha$-effect is the source of both poloidal and toroidal magnetic components. It works without a large scale flow. So, the planetary dynamos are believed to be of this kind [17], [35].

Figure 3.3 shows the comparative study of the observational with the theoretical solar butterfly diagram. Figure 3.3 (a) shows the latitude of sunspot appearance shown by black mark with time. The background shows the weak-large scale radial field on the photosphere. Figure 3.3 (b) is given by marking the locations of the eruption, "+" indicating the positive value of B at the bottom of the solar convective zone which erupts and "o" indicating the negative value. The sunspots eruptions are confined within $\pm 40^{\circ}$ and the butterfly diagrams have a shape similar to the observation [36], [37]. The weak radial field migrates poleward at higher latitudes, in similarity with observations. There is a phase relation between the sunspots and the weak diffuse field, which is the important aspects of observational data. The polar field changes from positive to negative at the time of a sunspot maximum corresponding to a negative toroidal field $\mathrm{B}$ at the base of the solar convection zone. This is clearly seen in theoretical results shown in Figure 3.3 (b).

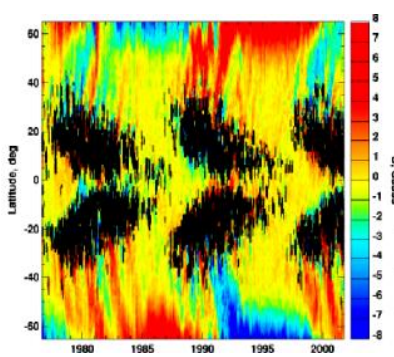

(a)

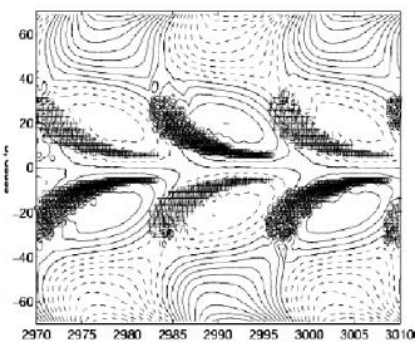

(b)
Figure 3.3: (a) The solar butterfly diagram depicting the latitude of sunspot appearance shown by black mark with time. The background shows the weak large-scale radial field on the photosphere. (b) Theoretical butterfly diagram of sunspots superposed on contours of constant $B_{r}$ at the solar surface in a time-latitude plot. The background shows contours of the diffuse radial field. [38]

\section{Conclusions}

The magnetic field of the Sun arises from the operation of a dynamo. The solar dynamo keeps the amplification of solar magnetic field through the action of fluid motions. The solar magnetic field manifests itself in the form of several transient and gradual solar activities. The evolution of Sun's magnetic field takes place on a wide range of spatial and temporal scales, including a main 11-year sunspot cycle. The 11year sunspot cycle controls the physical properties of the plasma flowing away from the Sun into the interplanetary space.

In particular, the explanation of the origin of the magnetic field of the Sun consists the fundamental problem of the theory of magnetohydrodynamics. The solar magnetic activity is responsible for many important solar phenomena, like solar explosions, the coronal mass ejections and heating the corona up to very high temperatures. They have important consequences for the geospace environment and Earth's climate system. These influences in the navigation systems of spacecraft orbiting the Earth, power grids, telecommunication system etc.

It is generally accepted that the strong toroidal magnetic field emerges through the solar surface 
Tiwari et al., Int. Ann. Sci.; Vol. 3 Issue 1, pp: 27-36, 2017

by the action of differential rotation at the base of the convective zone (tachocline) and formed the active regions and sunspots. Due to differential rotation of the Sun, there is the formation of the toroidal field from the poloidal field. But the generation of the poloidal field from the toroidal field due to the alpha effect is related to some form of turbulent helicity. It shows that both components of the fields may be sustained by the fluid flow. The mean-field electrodynamics explain the effects of the smallscale magnetic field and velocity field using transport coefficients. It describes the evolution of the large-scale field by averaging over the small-scale fluctuating field. The theoretical butterfly diagram obtained from the mean field dynamo i.e alpha-omega dynamo model is good agreement with the observed butterfly diagram.

\section{How to Cite this Article:}

B. R. Tiwari and M. Kumar, "An Insight into the Solar Dynamo Theory", International Annals of Science, vol. 3, no. 1, pp. 27-36, Dec. 2017. doi: 10.21467/ias.3.1.27-36

\section{References}

[1] J. Larmor, "How could a rotating body such as the Sun become a magnet?", Reports of the British Association, v. 87 , p. $159-160,1919$.

[2] R.H. Cranmer, M. Dikpati, A. Brandenburg, "The global solar dynamo", Space Science Review, 210, 367 - 395, 2017.

[3] A. Herzenberg, "Geomagnetic Dynamos", Philosophical Transactions of the Royal Society of London A", 250, 543-583, 1958.

[4] G. O. Roberts, "Spatially periodic dynamos", Philosophical Transactions of the Royal Society of London A", 266, 535-558, 1970.

[5] E.N. Parker, "Hydromagnetic Dynamo Models", The Astrophysical Journal, 122, 293, 1955.

[6] M. Steenbeck, F. Krause, K. -H. Radler, “A calculation of the mean electromotive force in an electrically conducting fluid in turbulent motion under the influence of Coriolis forces", Zeitschrift Naturforschung Teil A, v. 21, p. 369-376,1966.

[7] H.W. Babcock, "The topology of the Sun's magnetic field and the 22-year cycle", The Astrophysical Journal, 133, 572, 1961.

[8] R.B. Leighton, 1964, "Transport of magnetic fields on the Sun", The Astrophysical Journal, 140, 1547, 1964.

[9] R.B. Leighton, 1969, "A magneto-kinematic model of the solar cycle", The Astrophysical Journal, 156, 1969.

[10] M. Stix, "Comments on the solar dynamo", Astron. Astrophys, 37, 121 - 133, 1974.

[11] A. Courvoisier, "Dynamo Action and the Generation of Large-scale Magnetic Fields in Astrophysics", Thesis (Mathematics, PhD) University of Leeds, Leeds, 2006.
[12] S. M. Tobias, "The solar dynamo", Philosophical Transactions of Royal Society of London A, v. 360, p. 2741-2756, 2002.

[13] A. Brandenburg, K. Subramanian, "Astrophysical magnetic fields and nonlinear dynamo theory" Physics Reports, v. 417, 1-209, 2005.

[14] Agris Gailitis, Olgerts Lielausis, Sergej Dement'ev, Ernest Platacis, Arnis Cifersons, Gunter Gerbeth, Thoman Gundrum, Frank Stefani, Michael Christen, Heiko Hanel, and Gotthard Will, Physical Review Letters, v. 84, p. 4365, 2000.

[15] H. Alfven, "Remarks on the rotation of a magnetized sphere with application to the solar rotation", Ark.f.Mat.Astr.o.Fysik, 29B, No.2. 1942.

[16] A. R. Choudhuri, "The physics of fluids and plasmas: An Introduction for Astrophysicists", Cambridge University Press, 1998.

[17] M. Ossendrijver, "The solar dynamo", The Astronomy and Astrophysics Review, v. 11, p. 287- 367, 2003.

[18] T. G. Cowling, "The magnetic field of sunspots", MNRAS, v. 94, p. 34-48, 1933.

[19] M. Nunez, "The decay of axisymmetric magnetic fields: A review of cowling's theorem" SIAM REVIEW, v. 38, n.4, p. 553- 564, 1996.

[20] J. A. Bittencourt, "Fundamentals of Plasma Physics", [S.I]: Springer-Verlag, 2004.

[21] W.M. Elsasser, "Induction effects in terrestrial magnetism part I. theory", Physical Review, v. 69, p.106116, 1946

[22] M. R. E. Proctor, "An extension of the toroidal theorem", Geophysical and Astrophysical Fluid Dynamics, v. 98, p. 235-240, 2004.

[23] S. K. Solanki, B. Inhester, M. Schussler, "The solar magnetic fields", Reports on Progress In Physics, v. 69, p. 563-668, 2006.

[24] E. N. Parker, "The generation of magnetic fields in astrophysical bodies i. The dynamo equations" Astrophysical Journal, v.162, p. 665- 673, 1970.

[25] A. S. Monin, A. M. Yaglom, "Statistical Fluid Mechanics", vol. 1, MIT Press, Cambridge, 1973, ISBN:0-486-45891-1 (pbk)

[26] F. Krause, K. H. Radler, "Mean-Field Magnetohydrodynamics and Dynamo Theory", Pergamon Press, Elsevier, 1980.

[27] C. A. Jones, J. M. Thompson, S. M.Tobias, "The Solar Dynamo", Space Science Review, v.152, p. 591-616, 2010.

[28] H. K. Moffatt, "Magnetic field generation in electrically conducting fluids", Cambridge University Press 1978.

[29] Gorinsky, B., et al. "Evidence for the bilobal nature of diferric rabbit plasma transferrin." Nature 281.5727 (1979): 157-158.

[30] Krause, F., and K. H. Rädler. "Mean-field electrodynamics and dynamo theory." Akademie, Berlin, Germany (1980).

[31] Pipin, V.V. "Advances in Mean-Field Dynamo Theories" Solar and Astrophysical Dynamos and Magnetic Activity, Proceedings IAU Symposium No. 294, 2013

[32] P. H. Roberts, "Fundamentals of dynamo theory: in Lectures on Solar and Planetary Dynamos", Cambridge University Press,1-58,1994, ISBN: 9780521461429

[33] S. I. Vainshtein, F. Cattaneo, "Nonlinear restrictions on dynamo action" Astrophysical Journal, v. 393, p. 165, 1992. 
An Insight into the Solar Dynamo Theory

[34] N.O. Weiss, "Lectures on Solar and Planetary Dynamos", Cambridge University Press, 59, 1994.

[35] T. G. Cowling, "The present status of dynamo theory", Annual review of astronomy and astrophysics, v. 19, p. 115-135, 1981.

[36] E. N. Parker, "Cosmical Magnetic Fields: Their origin and their activity", Oxford University Press, 858, 1979.

[37] M. Stix, "The Sun: An Introduction”, Springer-Verlag, Berlin, 1989.

[38] P. Charbonneau, "Dynamo Models of the Solar Cycle", Living Review in Solar Physics, 7, 3, 2010.

Publish your research article in AIJR journals-

$\checkmark$ Online Submission and Tracking

$\checkmark$ Peer-Reviewed

$\checkmark$ Rapid decision

$\checkmark$ Immediate Publication after acceptance

$\checkmark$ Articles freely available online

$\checkmark \quad$ Retain full copyright of your article.

Submit your article at journals.aijr.in 